\title{
Variable Exponent Function Spaces related to a Sublinear Expectation
}

\author{
Bochi Xu \\ Department of Mathematics, Huaqiao University, Quanzhou 362021, China \\ Correspondence should be addressed to Bochi Xu; xubochi@126.com
}

Received 27 March 2019; Accepted 16 September 2019; Published 13 January 2020

Academic Editor: Shuangjie Peng

Copyright (c) 2020 Bochi Xu. This is an open access article distributed under the Creative Commons Attribution License, which permits unrestricted use, distribution, and reproduction in any medium, provided the original work is properly cited.

\begin{abstract}
In this paper, variable exponent function spaces $\mathbb{L}^{p^{(\cdot)},}, \mathbb{L}_{b}^{p^{(\cdot)}}$, and $\mathbb{L}_{c}^{p^{(\cdot)}}$ are introduced in the framework of sublinear expectation, and some basic and important properties of these spaces are given. A version of Kolmogorov's criterion on variable exponent function spaces is proved for continuous modification of stochastic processes.
\end{abstract}

\section{Introduction}

Variable exponent spaces are extensively applied in the study of some nonlinear problems in natural science and engineering. Basic properties of the spaces are first given by Kováčik and Rákosník in [1]. Some theories of variable exponent spaces can also be found in $[2,3]$. Harjulehto et al. present an overview of applications to differential equations with nonstandard growth in [4]. Diening et al. [5] summarize most of the existing literature of theory of variable exponent function spaces and applications to partial differential equations. In [6], Aoyama proves some important probability inequalities in variable exponent Lebesgue spaces.

Nonlinear expectations play an important role in the research of financial markets. One of the most important application is that a coherent risk measure(the basic theory about coherent risk measure can be found in [7]) is a sublinear expectation $\mathbb{E}: \mathscr{H} \rightarrow \mathbb{R}$ defined on $\mathscr{H}$, which is a linear space of finacial losses. In this paper, we are interested in behavior of sublinear expectation spaces with variable exponents. By the following representation theorem which can be found in Peng ([8], p. 4), we know that a sublinear expectation can be expressed as a supremum of linear expectations, i.e., there exists a family of linear expectations $\left\{E_{\theta}\right\}_{\theta \in \Theta}$ such that

$$
\mathbb{E}[X]=\sup _{\theta \in \Theta} E_{\theta}[X], \quad X \in \mathscr{H} .
$$

Thus, we consider the upper expectation only in this paper. Some other important theories about nonlinear expectations can be found in Peng's $[9,10]$.

The remainder of the paper is divided as follows: in Section 2, motivated by $\mathrm{Fu}$ [11] and Denis et al. [12], we first introduce $\mathbb{L}^{p(\cdot)}, \mathbb{L}_{b}^{p(\cdot)}$ and $\mathbb{L}_{c}^{p(\cdot)}$ and give some properties of these spaces. And Each element of $\|\cdot\|_{p(\cdot)}$-completion of $C_{b}(\Omega)$ has a quasi-continuous version is proved. In Section 3, applying the results of Section 2, we discuss a version of Kolmogorov's criterion for continuous modification of stochastic processes, which are in the variable exponent function space related to a sublinear expectation, after proving the situation under a linear expectation.

\section{Variable Exponent Function Spaces}

Let $\Omega$ be a complete metric space equipped with the distance d, $\mathscr{B}(\Omega)$ the Borel $\sigma$-algebra of $\Omega$ and $\mathscr{M}$ the collection of all probability measures on $(\Omega, \mathscr{B}(\Omega)$ ).

$L^{0}(\Omega)$ : the space of all $\mathscr{B}(\Omega)$-measurable real functions;

$B_{b}(\Omega)$ : all bounded functions in $L^{0}(\Omega)$;

$C_{b}(\Omega)$ : all continuous functions in $B_{b}(\Omega)$.

For a given subset $\mathscr{P} \subseteq \mathscr{M}$, we denote

$$
c(A):=\sup _{P \in \mathscr{P}} P(A), \quad A \in \mathscr{B}(\Omega) .
$$


Definition 1. A set $A$ is a polar if $c(A)=0$ and a property holds "quasi-surely" (q.s.) if it holds outside a polar set.

The upper expectation of $\mathscr{P}$ is defined as follows: for each $X \in L^{0}(\Omega)$ such that $E_{P}[X]$ exists for each $P \in \mathscr{P}$,

$$
\mathbb{E}[X]:=\sup _{P \in \mathscr{P}} E_{P}[X] .
$$

About $\mathbb{E}$, we know the following properties.

Theorem 2 (see Theorem 9 in [12]). The upper expectation $\mathbb{E}[\cdot]$ of family $\mathscr{P}$ is a sublinear expectation on $B_{b}(\Omega)$ as well as on $C_{b}(\Omega)$, i.e.,

(1) For all $X, Y$ inB $B_{b}(\Omega), X \geq Y, \mathbb{E}[X] \geq \mathbb{E}[Y]$.

(2) For all $X, Y \operatorname{inB}_{b}(\Omega), \mathbb{E}[X+Y] \leq \mathbb{E}[X]+\mathbb{E}[Y]$.

(3) For all $\lambda \geq 0, X \in B_{b}(\Omega), \mathbb{E}[\lambda X]=\lambda \mathbb{E}[X]$.

(4) For all $c \in \mathbb{R}, X \in B_{b}(\Omega), \mathbb{E}[X+c]=\mathbb{E}[X]+c$.

Let a $\mathscr{B}(\Omega)$-measurable real function $p: \Omega \rightarrow[1, \infty)$, be a variable exponent. In the space $L^{0}(\Omega)$, the moduli $\rho$ are defined by

$$
\begin{aligned}
\rho_{p(\cdot)}(X):=E\left[\left|X^{p}\right|\right] & =\sup _{P \in} E_{P}\left[\left|X^{p}\right|\right] \\
& =\sup _{P \in} \int_{\Omega}|X|(\omega) p(\omega) P(d \omega) .
\end{aligned}
$$

Definition 3. The space $\mathscr{L}^{p(\cdot)}$ is the set of $X \in L^{0}(\Omega)$ satisfying $\rho_{p(\cdot)}(X)<\infty$, and it is endowed with the Luxemburg norm:

$$
\|X\|_{p(\cdot)}=\inf \left\{\lambda>0: \rho_{p(\cdot)}\left(\frac{X}{\lambda}\right) \leq 1\right\} .
$$

We set $\mathscr{N}^{p(\cdot)}:=\left\{X \in L^{0}(\Omega): \rho(X)=0\right\}$, and denote $\mathbb{L}^{p(\cdot)}:=\mathscr{L}^{p(\cdot)} / \mathscr{N}^{p(\cdot)}$. As usual, we do not take care about the distinction between classes and their representatives.

Proposition 4. If the variable exponent $p$ satisfies $1<p^{-} \leq p(\omega) \leq p^{+}<\infty$, then the inequality

$$
\mathbb{E}[|X Y|] \leq\left(1+\frac{1}{p^{-}}-\frac{1}{p^{+}}\right)\|X\|_{p(\cdot)}\|Y\|_{p^{\prime}(\cdot)}
$$

holds for every $X \in \mathbb{L}^{p(\cdot)}$ and $Y \in \mathbb{L}^{p^{\prime}(\cdot)}$, where $1 / p(\omega)+$ $1 / p^{\prime}(\omega)=1$.

Proof. By Young inequality, we have

$$
\frac{|X|}{\|X\|_{p(\cdot)}} \frac{|Y|}{\|Y\|_{p^{\prime}(\cdot)}} \leq \frac{1}{p(\omega)}\left(\frac{|X|}{\|X\|_{p(\cdot)}}\right)^{p}+\frac{1}{p^{\prime}(\omega)}\left(\frac{|Y|}{\|Y\|_{p^{\prime}(\cdot)}}\right)^{p^{\prime}} .
$$

By the monotonicity, sub-additivity and positive homogeneity of $\mathbb{E}$, and the property of the norm, we have

$$
\begin{aligned}
& \mathbb{E}\left[\frac{|X|}{\|X\|_{p(\cdot)}} \frac{|Y|}{\|Y\|_{p^{\prime}(\cdot)}}\right] \\
& \leq \mathbb{E}\left[\frac{1}{p(\omega)}\left(\frac{|X|}{\|X\|_{p(\cdot)}}\right)^{p}+\frac{1}{p^{\prime}(\omega)}\left(\frac{|Y|}{\|Y\|_{p^{\prime}(\cdot)}}\right)^{p^{\prime}}\right] \\
& \quad \leq \mathbb{E}\left[\frac{1}{p(\omega)}\left(\frac{|X|}{\|X\|_{p(\cdot)}}\right)^{p}\right]+\mathbb{E}\left[\frac{1}{p^{\prime}(\omega)}\left(\frac{|Y|}{\|Y\|_{p^{\prime}(\cdot)}}\right)^{p^{\prime}}\right] \\
& \quad \leq \frac{1}{p^{-}}\left[\left(\frac{|X|}{\|X\|_{p(\cdot)}}\right)^{p}\right]+\left(1-\frac{1}{p^{+}}\right) \mathbb{E}\left[\left(\frac{|Y|}{\|Y\|_{p^{\prime}(\cdot)}}\right)^{p^{\prime}}\right] \\
& \quad \leq 1+\frac{1}{p^{-}}-\frac{1}{p^{+}} .
\end{aligned}
$$

Thus, the inequality follows.

Proposition 5. Suppose that the variable exponent $p$ satisfies $1<p^{-} \leq p(\omega) \leq p^{+}<\infty$. If $X, X_{k} \in \mathbb{L}^{p(\cdot)}$, then

(1) If $\|X\|_{p(\cdot)} \geq 1$, then $\|X\|_{p(\cdot)}^{p^{-}} \leq \rho_{p(\cdot)}(X) \leq\|X\|_{p(\cdot)}^{p^{+}}$.

(2) If $\|X\|_{p(\cdot)} \leq 1$, then $\|X\|_{p(\cdot)}^{p+} \leq \rho_{p(\cdot)}(X) \leq\|X\|_{p(\cdot)}^{p}$.

(3) $\lim _{k \rightarrow \infty}\left\|X_{k}\right\|_{p(\cdot)}=0$, if and only if $\lim _{k \rightarrow \infty} \rho_{p(\cdot)}\left(X_{k}\right)=0$

(4) $\lim _{k \rightarrow \infty}\left\|X_{k}\right\|_{p(\cdot)}=\infty$, if and only if $\lim _{k \rightarrow \infty} \rho_{p(\cdot)}$ $\left(X_{k}\right)=\infty$.

In particular, the linear expectation $E_{P}$ also follows this proposition.

Proof. (1) By $\|X\| \mathrm{P}_{p(\cdot)} \geq 1$ and the definition of the norm,

$$
\mathbb{E}\left[\frac{|X|^{p}}{\|X\|_{p(\cdot)}^{p^{+}}}\right] \leq \mathbb{E}\left[\left(\frac{|X|}{\|X\|_{p(\cdot)}}\right)^{p}\right] \leq 1 .
$$

Thus, $\rho_{p(\cdot)}(X) \leq\|X\|_{p(\cdot)}^{p^{+}}$. As

$$
\left\|X_{p(\cdot)}^{p^{-} / p} \leq\right\| X \|_{p(\cdot)},
$$

we also have

$$
\mathbb{E}\left[\left(\frac{|X|}{\|X\|_{p(\cdot)}^{p^{-} / p}}\right)^{p}\right] \geq 1 .
$$

That is to say $\|X\|_{p(\cdot)}^{p^{-}} \leq \rho_{p(\cdot)}(X)$. The proof is completed. (2) can be easily proved in the similar method. By (1) and (2), we can easily get (3) and (4).

Proposition 6. Suppose that the variable exponent $p$ satisfies $1<p^{-} \leq p(\omega) \leq p^{+}<\infty$. Let $X, X_{k} \in \mathbb{L}^{p(\cdot)}$. Then for each $\alpha>0$

$$
c(\{|X|>\alpha\}) \leq\left(\alpha^{-p^{-}} \vee \alpha^{-p^{+}}\right) \mathbb{E}[|X p|] .
$$

Proof. For each $P \in \mathscr{P}$, by Markov inequality, we have 


$$
P\left(\left\{\left|\frac{X}{\alpha}\right|^{p}>1\right\}\right) \leq E_{P}\left[\left|\frac{X}{\alpha}\right|^{p}\right] .
$$

Take the supremum in $\mathscr{P}$ both sides,

$$
c\left(\left\{\left|\frac{X}{\alpha}\right|^{p}>1\right\}\right) \leq \mathbb{E}\left[\left|\frac{X}{\alpha}\right|^{p}\right]
$$

Thus,

$$
c(\{|X|>\alpha\}) \leq \mathbb{E}\left[\left|\frac{X}{\alpha}\right|^{p}\right] \leq\left(\alpha^{-p^{-}} \vee \alpha^{-p^{+}}\right) \mathbb{E}\left[|X|^{p}\right] .
$$

Lemma 7 (Proposition 17 in [12]). Let $p \in(0, \infty]$ and $\left\{X_{n}\right\}$ be a sequence in $\mathbb{L}^{p}$ which converges to $X$ in $\mathbb{L}^{p}$. Then there exists a subsequence $\left\{X_{n_{k}}\right\}$ which converges to $X$ quasi-surely in the sense that it converges to X outside a polar set.

Proposition 8. If the variable exponent $p$ satisfies $1<p^{-} \leq p(\omega) \leq p^{+}<\infty . \mathbb{L}^{p(\cdot)}$ is a Banach space.

Proof. Let $\left\{X_{n}\right\}$ be a Cauchy sequence in $\mathbb{L}^{p(\cdot)}$. Then, by Proposition 4,

$$
\mathbb{E}\left[\left|X_{m}-X_{n}\right|\right] \leq C\left\|X_{m}-X_{n}\right\|_{p(\cdot)},
$$

where $C$ is a constant. That is to say $\left\{X_{n}\right\}$ is a Cauchy sequence in $\mathbb{L}^{1}$. By Proposition 14 in [12], $\mathbb{L}^{1}$ is a Banach Space. Thus, $\left\{X_{n}\right\}$ converges in $\mathbb{L}^{1}$. Suppose that $X_{n} \rightarrow X, X \in \mathbb{L}^{1}$ and further by Lemma 7, we suppose $X_{n} \rightarrow X$ quasi-surely (subtracting a subsequence if necessary). For each $0<\varepsilon<1$, there exists $n_{0}$ such that $\left\|X_{m}-X_{n}\right\|_{p(\cdot)}<\varepsilon$ for $m, n \geq n_{0}$. Fix $n$, by Fatou's lemma

$$
\begin{aligned}
\mathbb{E}\left[\left(\frac{\left|X_{n}-X\right|}{\varepsilon}\right)^{p}\right] & =\sup _{P \in \mathscr{P}} E_{P}\left[\left(\frac{\left|X_{n}-X\right|}{\varepsilon}\right)^{p}\right] \\
& \leq \sup _{P \in \mathscr{P}} \liminf _{m \rightarrow \infty} E_{P}\left[\left(\frac{\left|X_{n}-X_{m}\right|}{\varepsilon}\right)^{p}\right] \\
& \leq \liminf _{m \rightarrow \infty} \mathbb{E}\left[\left(\frac{\left|X_{n}-X_{m}\right|}{\varepsilon}\right)^{p}\right] \leq 1 .
\end{aligned}
$$

Thus, $\left\|X_{n}-X\right\|_{p(\cdot)} \leq \varepsilon$, and further $\rho_{p(\cdot)}\left(X_{n}-X\right) \leq\left\|X_{n}-X\right\|_{p(\cdot)}^{p^{-}}$ $\leq \varepsilon^{p^{-}}$, that is to say, $X_{n}-X \in \mathbb{L}^{p(\cdot)}$, and $X \in \mathbb{L}^{p(\cdot)}$. The proof is completed.

We denote by $\mathbb{L}_{b}^{p(\cdot)}$ the completion of $B_{b}(\Omega)$ and by $\mathbb{L}_{c}^{p(\cdot)}$ the completion of $C_{b}(\Omega)$. By Proposition 8 , we have $\mathbb{L}_{c}^{p(\cdot)} \subset \mathbb{L}_{b}^{p(\cdot)} \subset \mathbb{L}^{p(\cdot)}$ for $1<p^{-} \leq p(\omega) \leq p^{+}<\infty$.

Proposition 9. Suppose that the variable exponent $p$ satisfies $1<p^{-} \leq p(\omega) \leq p^{+}<\infty$, then

$$
\mathbb{L}_{b}^{p(\cdot)}=\left\{X \in \mathbb{L}^{p(\cdot)}: \lim _{n \rightarrow \infty} \mathbb{E}\left[|X|^{p} 1_{\{|X|>n\}}\right]=0\right\} .
$$

Proof. Wedenote $J_{p(\cdot)}=\left\{X \in \mathbb{L}^{p(\cdot)}: \lim _{n \rightarrow \infty} \mathbb{E}\left[|X|^{p} 1_{\{|X|>n\}}\right]=0\right\}$. For each $X \in J_{p(\cdot)}$ let $X_{n}=(X \wedge n) \vee(-n) \in B_{b}(\Omega)$. We have

$$
\rho_{p(\cdot)}\left(X-X_{n}\right) \leq \mathbb{E}\left[|X|^{p} 1_{\{|X|>n\}}\right],
$$

so $\lim _{n \rightarrow \infty} \rho_{p(\cdot)}\left(X-X_{n}\right)=0$. Thus, $X \in \mathbb{L}_{b}^{p(\cdot)}$. On the other hand, for any $X \in \mathbb{L}_{b}^{p(\cdot)}$, we can find a sequence $\left\{Y_{n}\right\} \subset B_{b}(\Omega)$ such that $\lim _{n \rightarrow \infty} \rho_{p(\cdot)}\left(X-Y_{n}\right)=0$. Let $y_{n}=\sup _{\omega \in \Omega}\left|Y_{n}(\omega)\right|$ and $X_{n}=\left(X \wedge y_{n}\right) \vee\left(-y_{n}\right)$. Since $\left|X-X_{n}\right| \leq\left|X-Y_{n}\right|$, we have $\lim _{n \rightarrow \infty} \rho_{p(\cdot)}\left(X-X_{n}\right)=0$. This implies that for any sequence $\left\{\alpha_{n}\right\}$ such that $\alpha_{n} \rightarrow \infty$ as $n \rightarrow \infty, \lim _{n \rightarrow \infty} \rho_{p(\cdot)}\left(X-\left(X \wedge \alpha_{n}\right)\right.$ $\left.\vee\left(-\alpha_{n}\right)\right)=0$. And for all $n \in \mathbb{N}$,

$\mathbb{E}\left[|X|^{p} 1_{\{|X|>n\}}\right]<2^{p^{+}-1} \mathbb{E}\left[(|X|-n)^{p} 1_{\{|X|>n\}}\right]+\mathbb{E}\left[2^{p-1} n^{p} 1_{\{|X|>n\}}\right]$.

For the first term of right hand side, we have

$\operatorname{Lim}_{n \rightarrow \infty} \mathbb{E}\left[(|X|-n) p 1_{\{|X|>n\}}\right]=\lim _{n \rightarrow \infty} \rho_{p(\cdot)}(X-(X \wedge n) \vee(-n))=0$

For the second term,

$\frac{n^{p}}{2^{p}} 1_{\{|X|>n\}} \leq\left(|X|-\frac{n}{2}\right)^{p} 1_{\{|X|>n\}} \leq\left(|X|-\frac{n}{2}\right)^{p} 1_{\{|X|>n / 2\}}$,

so

$$
\begin{aligned}
\mathbb{E}\left[2^{p-1} n^{p} 1_{\{|X|<n\}}\right] & =\mathbb{E}\left[2^{2 p-1} \frac{n^{p}}{2^{p}} 1_{\{|X|<n\}}\right] \\
& \leq \mathbb{E}\left[2^{2 p-1}\left(|X|-\frac{n}{2}\right)^{p} 1_{\{|X|<n / 2\}}\right] \\
& \leq 2^{2 p^{+}-1} \mathbb{E}\left[(|X|-(n / 2))^{p} 1^{\{|X|<n / 2\}}\right],
\end{aligned}
$$

and $\lim _{n \rightarrow \infty} \mathbb{E}\left[2^{p-1} n^{p} 1_{\{|X|<n\}}\right]=0$. Thus, $X \in J_{p(\cdot)}$.

Proposition 10. If the variable exponent $p$ satisfies $1<p^{-} \leq p(\omega) \leq p^{+}<\infty$, let $X \in \mathbb{L}_{b}^{p(\cdot)}$, then for each $\varepsilon>0$, there exists a $\delta>0$, such that for all $A \in \mathscr{B}(\Omega)$ with $c(A) \leq \delta$, we have $\mathbb{E}\left[|X|^{p} 1_{A}\right] \leq \varepsilon$.

Proof. For each $\varepsilon>0$, by Proposition 9, there exists $N>0$ such that $\mathbb{E}\left[|X|^{p} 1_{\{|X|<n\}}\right] \leq \varepsilon / 2$. Set $\delta=\varepsilon / 2 N^{p^{+}}$, then for $A \in \mathscr{B}(\Omega)$ with $c(A) \leq \delta$, we have.

$$
\begin{aligned}
\mathbb{E}\left[|X|^{p} 1_{A}\right] & \leq \mathbb{E}\left[|X|^{p} 1_{A} 1_{\{|X|<N\}}\right]+\mathbb{E}\left[|X|^{p} 1_{A} 1_{\{|X| \leq N\}}\right] \\
& \leq \mathbb{E}\left[|X|^{p} 1_{\{|X|<N\}}\right]+N^{p^{+}} c(A) \leq \varepsilon .
\end{aligned}
$$

Definition 11. A mapping $X$ on $\Omega$ with values in topological space is said to be quasi-continuous (q.c.) if $\forall \varepsilon>0$, there exists an open set $O$ with $c(O)<\varepsilon$ such that $\left.X\right|_{O^{c}}$ is continuous.

Definition 12. We say that $X: \Omega \rightarrow \mathbb{R}$ has a quasi-continuous version if there exists a quasi-continuous $Y: \Omega \rightarrow \mathbb{R}$ with $X=Y$ q.s.

Proposition 13. If the variable exponent $p$ satisfies $1<p^{-} \leq p(\omega) \leq p^{+}<\infty$. Then each element in $\mathbb{L}_{c}^{p^{(\cdot)}}$ has a quasi-continuous version. 
Proof. For each $X \in \mathbb{L}_{c}^{p(\cdot)}$, there exists $\left\{X_{n}\right\} \subset C_{b}(\Omega)$ such that $X_{n} \rightarrow X$ in $\mathbb{L}_{c}^{p(\cdot)}$. Let us choose a subsequence $\left\{X_{n_{k}}\right\}$ such that

$$
\rho_{p(\cdot)}\left(X_{n_{k+1}}-X_{n_{k}}\right) \leq 2^{-2 k}, \quad \forall k \geq 1,
$$

and set for all $k$,

$$
A_{k}=\bigcup_{i=k}^{\infty}\left\{\left|X_{n_{i+1}}-X_{n_{i}}\right|>2^{-i / p^{+}}\right\} .
$$

Because of the subadditivity property and Proposition 6,

$$
\begin{aligned}
c\left(A_{k}\right) & \leq \sum_{i=k}^{\infty} c\left(\left|X_{n_{i+1}}-X_{n_{i}}\right|<2^{-i / p^{+}}\right) \\
& \leq \sum_{i=k}^{\infty} 2^{i} \mathbb{E}\left[\left|X_{n_{i+1}}-X_{n_{i}}\right| p\right] \leq \sum_{i=k}^{\infty} 2^{-i}=2^{-k+1} .
\end{aligned}
$$

Thus, $\lim _{k \rightarrow \infty} c\left(A_{k}\right)=0$, so the Borel set $A=\bigcap_{k=1}^{\infty} A_{k}$ is polar. As each $X_{n_{k}}$ is continuous, for all $k \geq 1, A_{k}$ is an open set. And for all $k,\left\{X_{n_{i}}\right\}$ converges uniformly on $A_{k}^{c}$ so that the limit is continuous on each $A_{k}^{c}$.

Proposition 14. Suppose that the variable exponent $p$ satisfies $1<p^{-} \leq p(\omega) \leq p^{+}<\infty$, then

$$
\begin{aligned}
\mathbb{L}_{c}^{p(\cdot)}= & \left\{X \in \mathbb{L}^{p(\cdot)}:\right. \text { X has a quasicontinuous version, } \\
& \text { and } \left.\lim _{n \rightarrow \infty} \mathbb{E}\left[|X|^{p} 1_{\{|X|<n\}}\right]=0\right\}
\end{aligned}
$$

Proof. Let

$$
\begin{aligned}
J_{p(\cdot)}= & \left\{X \in \mathbb{L}^{p(\cdot)}: X\right. \text { has a quasicontinuous version, } \\
& \text { and } \left.\lim _{n \rightarrow \infty} \mathbb{E}\left[|X|^{p} 1_{\{|X|<n\}}\right]=0\right\} .
\end{aligned}
$$

For each $X \in \mathbb{L}_{c}^{p(\cdot)}, X$ has a quasi-continuous version by Proposition 13. Since $X \in \mathbb{L}_{c}^{p(\cdot)} \subset \mathbb{L}_{b}^{p(\cdot)}$, by Proposition 9 we have $\lim _{n \rightarrow \infty} \mathbb{E}\left[|X|^{p} 1_{\{|X|>n\}}\right]=0$. Thus, $X \in J_{p(\cdot) \cdot}$.

On the other hand, Let $X \in J_{p(\cdot)}$ be quasi-continuous. For all $n \in \mathbb{N}$, define

$$
Y_{n}=(X \wedge n) \vee(-n)
$$

Since $\lim _{n \rightarrow \infty} \mathbb{E}\left[|X|^{p} 1_{\{|X|>n\}}\right]=0$ and

$$
\begin{aligned}
E\left[\left|X-Y_{n}\right|^{p}\right] & \leq E\left[\left|X-Y_{n}\right|^{p} 1_{\{|X|<n\}}\right]+E\left[\left|X-Y_{n}\right|^{p} 1_{\{|X| \leq n\}}\right] \\
& \leq E\left[|X|^{p} 1_{\{|X|<n\}}\right]
\end{aligned}
$$

we have $\lim _{n \rightarrow \infty} \mathbb{E}\left[\left|X-Y_{n}\right|^{p}\right]=0$.

For all $_{n}^{\infty} \in \mathbb{N}, Y_{n}$ is quasi-continuous, so there exists a closed set $F_{n}$ such that $c\left(F_{n}^{c}\right)<1 / n^{p^{+}+1}$ and $Y_{n}$ is continuous on $F_{n}$. By Tietze's extension theorem there exists $Z_{n} \in C_{b}(\Omega)$ such that $\left|Z_{n}\right| \leq n$ and $Z_{n}=Y_{n}$ on $F_{n}$. Then, we have

$$
\begin{aligned}
E\left[\left|Y_{n}-Z_{n}\right|^{p}\right] & \leq E\left[\left|Y_{n}-Z_{n}\right|^{p} 1_{F_{n}}\right]+E\left[\left|Y_{n}-Z_{n}\right|^{p} 1_{F_{n}^{c}}\right] \\
& \leq E\left[(2 n)^{p} 1_{F_{n}^{c}}\right] \leq(2 n)^{p^{+}} c\left(F_{n}^{c}\right) \leq \frac{2^{p^{+}}}{n} .
\end{aligned}
$$

Thus,

$$
\begin{aligned}
E\left[X-\left.Z_{n}\right|^{p}\right] & \leq 2^{p^{+}-1}\left(E\left[\left|X-Y_{n}\right|^{p}\right]+E\left[\left|Y_{n}-Z_{n}\right|^{p}\right]\right) \\
& \leq 2^{p^{+}-1} E\left[\left|X-Y_{n}\right|^{p}\right]+\frac{2^{2 p^{+}-1}}{n}
\end{aligned}
$$

As a consequence, $X \in \mathbb{L}_{c}^{p(\cdot)}$.

Proposition 15. Suppose that $X: \Omega \rightarrow \mathbb{R}$ has a quasicontinuous version and that there exists a function $f: \mathbb{R}^{+} \rightarrow \mathbb{R}^{+}$ satisfying $\lim _{n \rightarrow \infty} f(t) / t^{p}=\infty$ uniformly on $\Omega$ and $\mathbb{E}[f(|X|)]<\infty$. Then $X \in \mathbb{L}_{c}^{p(\cdot)}$, where $1<p^{-} \leq p(\omega) \leq p^{+}<\infty$.

Proof. For each $\varepsilon>0$, there exists $N>0$ which is independent of $\omega$ such that for all $t \geq N$,

$$
\frac{f(t)}{t^{p}} \geq \frac{1}{\varepsilon}
$$

So

$\mathbb{E}\left[|X|^{p} 1_{\{|X|>N\}}\right] \leq \varepsilon \mathbb{E}\left[f(|X|) 1_{\{|X|>N\}} \leq \varepsilon \mathbb{E}[f(|X|)]\right]$.

Thus, $\lim _{N \rightarrow \infty} \mathbb{E}\left[|X|^{p} 1_{\{|X|>N\}}\right]=0$.

\section{Kolmogorov's Criterion on Variable Exponent Function Spaces}

Definition 16. Let $I$ be a set of indices. $\left\{X_{t}\right\}_{t \in I}$ and $\left\{Y_{t}\right\}_{t \in I}$ be two processes indexed by $I$. We say that $Y$ is a quasimodification of $X$ if for all $t \in I X_{t}=Y_{t}$ q.s.

To prove a Kolmogorov's criterion for a process indexed by $\mathbb{R}^{d}$ with $d \in \mathbb{N}$ on variable exponent function spaces, we give the following lemma first.

Lemma 17. Let $1<p^{-} \leq p(\omega) \leq p^{+}<\infty$ and $\left\{X_{t}\right\}_{t \in[0,1]^{d}}$ be a process such that for all $t \in[0,1]^{d}, X_{t} \in \mathbb{L}^{p(\cdot)}$. Assume that for a fixed $P \in \mathscr{P}$ there exist positive constants $c$ and $\varepsilon$ such that

$$
E_{P}\left[\left|X_{t}-X_{s}\right| p\right] \leq c|t-s| d+\varepsilon
$$

Then $X$ admit a modification $\tilde{X}$ such that

$$
E_{P}\left[\left(\sup _{s \neq t} \frac{\left|\tilde{X}_{t}-\tilde{X}_{s}\right|}{|t-s|^{\alpha}}\right)^{p}\right]<\infty,
$$

for every $\alpha \in\left[0, \varepsilon / p^{+}\right]$

Proof. Fix $P \in \mathscr{P}$. For $m \in \mathbb{N}$, define the set of dyadic points in $[0,1]^{d}$ :

$$
D_{m}:=\left\{\left(\frac{i_{1}}{2^{m}}, \cdots, \frac{i_{d}}{2^{m}}\right): i_{1} \cdots, i_{d} \in\left\{0,1, \cdots, 2^{m}\right\}\right\},
$$

and $D=\cup_{m} D_{m}$. Set $\Delta_{m}=\left\{(s, t):|s-t|=2^{-m}, s, t \in D_{m}\right\}$, and there are fewer than $2^{(m+l) d}$ such pairs in $\Delta_{m}$. For $s, t \in D$, we say that $s \leq t$ if each component of $s$ is less than or equal to the corresponding component of $t$.

Set $K_{i}=\sup \left|X_{s}-X_{t}\right|$. And by the hypothesis in the lemma, 


$$
E_{P}\left[K_{i}^{p}\right] \leq \sum_{(s, t) \in \Delta_{i}} E_{P}\left[\left|X_{s},-, X_{t}\right|^{p}\right] \leq 2^{(i+1) d} c 2^{-i(d+\varepsilon)}=c 2^{d} 2^{-i \varepsilon}
$$

It is easy to see $c 2^{d} 2^{-i \varepsilon}<1$ from some $i_{0}$ on.

For $s, t \in D$, there exists increasing sequences $\left\{s_{n}\right\} \subset D$ and $\left\{t_{n}\right\} \subset D$ such that $s_{n}, t_{n} \in D_{n}, s_{n} \leq s, t_{n} \leq t$ and $s_{n}=s, t_{n}=t$ from some $n$ on.

Let now $s, t \in D$ and $|s-t| \leq 2^{-m}$, and either $s_{m}=t_{m}$ or $\left(s_{m}, t_{m}\right) \in \Delta_{m}$ and in any case,

$$
X_{s}-X_{t}=\sum_{i=m}^{\infty}\left(X_{s_{i+1}}-X_{s_{i}}\right)+X_{s_{m}}-X_{t_{m}}+\sum_{i=m}^{\infty}\left(X_{t_{i}}-X_{t_{i+1}}\right)
$$

where the series are actually finite sums. It follows that

$$
\left|X_{s}-X_{t}\right| \leq K_{m}+2 \sum_{i=m+1}^{\infty} K_{i} \leq 2 \sum_{i=m}^{\infty} K_{i} .
$$

Thus, setting $M_{\alpha}=\sup _{s, t \in D, s \neq t}\left|X_{s}-X_{t}\right| /|s-t|^{\alpha}$, we have

$$
\begin{gathered}
M_{\alpha} \leq \sup _{m \in \mathbb{N}}\left\{2^{(m+1) \alpha} \sup _{s, t \in D, s \neq t,|s-t| \leq 2^{-m}}\left|X_{s}-X_{t}\right|\right\} \\
\leq \sup _{m \in \mathbb{N}}\left\{2^{(m+1) \alpha+1} \sum_{i=m}^{\infty} K_{i}\right\} \leq 2^{\alpha+1} \sum_{i=0}^{\infty} 2^{i \alpha} K_{i} .
\end{gathered}
$$

As

$$
\begin{aligned}
K_{i p(\cdot)} & \leq \max \left\{\left(E_{P}\left[\left|K_{i}\right| p\right]\right)^{1 / p^{-}},\left(E_{P}\left[\left|K_{i}\right| p\right]\right)^{1 / p^{+}}\right\} \\
& \leq \max \left\{\left(c 2^{d} 2^{-i \varepsilon}\right)^{1 / p^{-}},\left(c 2^{d} 2^{-i \varepsilon}\right)^{1 / p^{+}}\right\},
\end{aligned}
$$

for every $\alpha \in\left[0, \varepsilon / p^{+}\right]$, we have

$$
\begin{aligned}
\left\|M_{\alpha}\right\|_{p(\cdot)} & \leq 2^{\alpha+1} \sum_{i=0}^{\infty} 2^{i \alpha}\left\|K_{i}\right\|_{p(\cdot)} \\
& \leq 2^{\alpha+1}\left(\sum_{i=0}^{i_{0}-1} 2^{i \alpha}\left(c 2^{d} 2^{-i \varepsilon}\right)^{1 / p^{-}}+\sum_{i=i_{0}}^{\infty} 2^{i \alpha}\left(c 2^{d} 2^{-i \varepsilon}\right)^{1 / p^{+}}\right) \\
& \left.\leq C_{\alpha, p^{-}, \varepsilon}+C_{d, \alpha, p^{+}, \varepsilon} \sum_{i=i_{0}}^{\infty} 2^{i\left(\alpha-\varepsilon / p^{+}\right.}\right)<\infty
\end{aligned}
$$

where $\alpha \in\left[0, \varepsilon / p^{+}\right)$. It follows in particular that for almost every $\omega, X$ is uniformly continuous on $D$ and it makes sense to set

$$
\tilde{X}_{t}(\omega)=\lim _{s \rightarrow t, s \in D} X_{s}(\omega)
$$

By Fatou's lemma and the hypothesis,

$$
E_{P}\left[\left|\tilde{X}_{t}-X_{t}\right|^{p}\right] \leq \liminf _{s \rightarrow t} E_{P}\left[\left|X_{s}-X_{t}\right|^{p}\right] \leq \liminf _{s \rightarrow t} c|t-s|^{d+\varepsilon},
$$

so, $\tilde{X}_{t}=X_{t}$ a.s. and $\tilde{X}_{t}$ is a modification.

Theorem 18. Let $1<p^{-} \leq p(\omega) \leq p^{+}<\infty$ and $\left\{X_{t}\right\}_{t \in[0,1]^{d}}$ be a process such that for all $t \in[0,1]^{d}, X_{t} \in \mathbb{L}^{p(\cdot)}$. Assume that there exists a positive constants $c$ and $\varepsilon$ such that

$$
E\left[\left|X_{t}-X_{s}\right|^{p}\right] \leq c|t-s|^{d+\varepsilon}
$$

Then $X$ admit a modification $\tilde{X}$ such that

$$
E\left[\left(\sup _{s \neq t} \frac{\left|\tilde{X}_{t}-\tilde{X}_{s}\right|}{|t-s| \alpha} p\right)\right]<\infty
$$

for every $\alpha \in\left[0, \varepsilon / p^{+}\right)$.

Proof. Let set $D$ the same as the proof of Lemma 17. We set.

$$
M_{\alpha}=\sup _{s, t \in D, s \neq t} \frac{\left|X_{s}-X_{t}\right|}{|s-t|^{\alpha}}
$$

where $\alpha \in\left[0, \varepsilon / p^{+}\right]$. By Lemma 17 , we know that for any $P \in \mathscr{P}, E_{P}\left[M^{p}\right]$ is finite and uniformly bounded with respect to $P$ so that

$$
\mathbb{E}\left[M^{P}\right]=\sup _{P \in \mathscr{P}} E_{P}\left[M^{P}\right]<\infty .
$$

Thus, $X$ is uniformly continuous on $D$ q.s. and set

$$
\tilde{X}_{t}(\omega)=\lim _{s \rightarrow t, s \in D} X_{s}(\omega), \quad t \in[0,1]^{d}
$$

In the similar way in Lemma $17, \tilde{X}_{t}=X_{t}$ q.s. and $\tilde{X}_{t}$ is a modification.

\section{Data Availability}

No data were used to support this study.

\section{Conflicts of Interest}

The authors declare that they have no conflicts of interest.

\section{Acknowledgments}

The author gratefully acknowledges the support from Fujian Provincial Natural Science Foundation (2016J05008).

\section{References}

[1] O. Kováčik and J. Rákosník, "On spaces $\mathrm{L}^{\mathrm{p}(\mathrm{x})}$ and $\mathrm{W}^{\mathrm{k}, \mathrm{p}(\mathrm{x}) \text {," }}$ Czechoslovak Mathematical Journal, vol. 41, pp. 592-618, 1991.

[2] L. Diening, "Maximal function on generalized lebesgue spaces

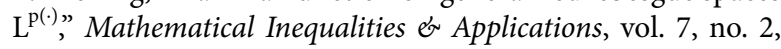
pp. 245-253, 1998.

[3] T. Futamura, Y. Mizuta, and T. Shimomura, "Sobolev embeddings for variable exponent riesz potentials on metric spaces," Annales Academioe Scientiarum Fennico Mathematica, vol. 31, pp. 495-522, 2006.

[4] P. Harjulehto, P. Hästö, U. V. Le, and M. Nuortio, "Overview of differential equations with non-standard growth," Nonlinear Analysis: Theory, Methods \& Applications, vol. 72, no. 12, pp. 4551-4574, 2010. 
[5] L. Diening, P. Harjulehto, P. Hästö, and M. Ruzicka, Lebesgue and Sobolev Spaces with Variable Exponents, Springer, 2010.

[6] H. Aoyama, "Lebesgue spaces with variable on a probability space," Hiroshima Mathematical Journal, vol. 39, no. 2, pp. 207-216, 2009.

[7] P. Artzner, F. Delbaen, J.-M. Eber, and D. Heath, "Coherent measures of risk," Mathematical Finance, vol. 9, no. 3, pp. 203228, 1999.

[8] S. Peng, "Nonlinear expectations and stochastic calculus under uncertainty," 2010, http://arxiv.org/pdf/1002.4546v1.pdf.

[9] S. Peng, "Nonlinear expectations and nonlinear markov chains," Chinese Annals of Mathematics, vol. 26B, no. 2, pp. 159-184, 2005.

[10] S. Peng, "Multi-dimensional g-brownian motion and related stochastic calculus under g-expectation," Stochastic Processes and their Applications, vol. 118, pp. 2223-2253, 2008.

[11] Y. Q. Fu, "Weak solution for obstacle problem with variable growth," Nonlinear Analysis, vol. 59, no. 3, pp. 371-383, 2004.

[12] L. Denis, M. Hu, and S. Peng, "Function spaces and capacity related to a sublinear expectation: application to g-brownian motion paths," Potential Analysis, vol. 34, no. 2, pp. 139-161, 2011.

[13] D. Revuz and M. Yor, "Continuous Martingales And Brownian Motion," Grundlehren der Mathematischen Wissenschaften, vol. 293, Springer-Verlag, Berlin, 3rd edition, 1999. 


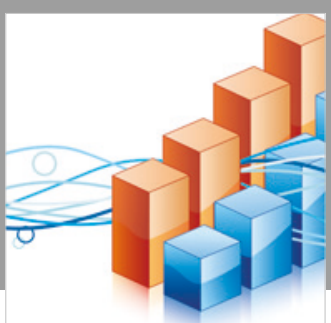

Advances in

Operations Research

\section{-n-m}
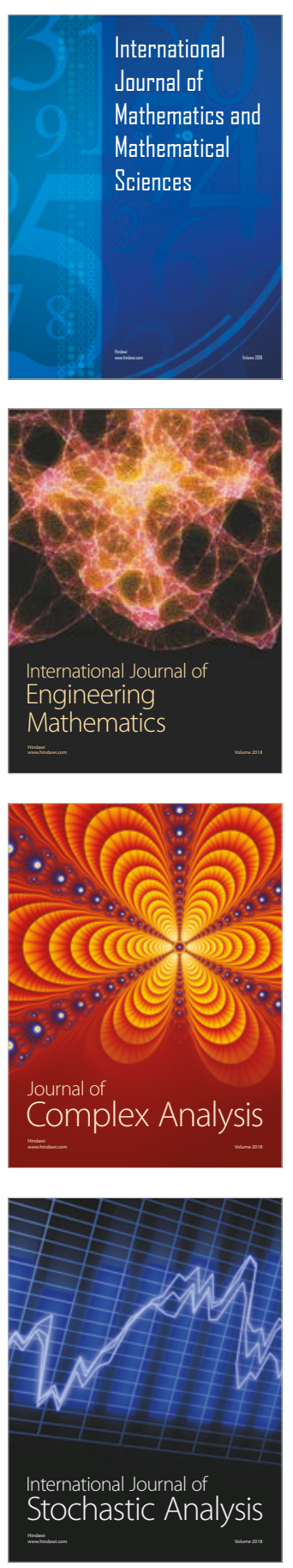
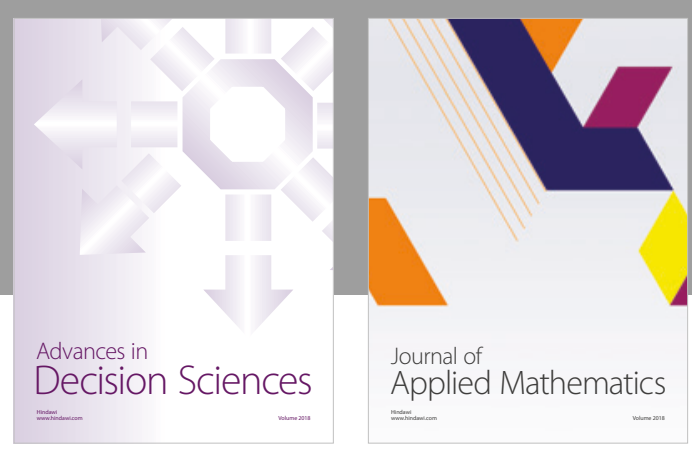

Journal of

Applied Mathematics
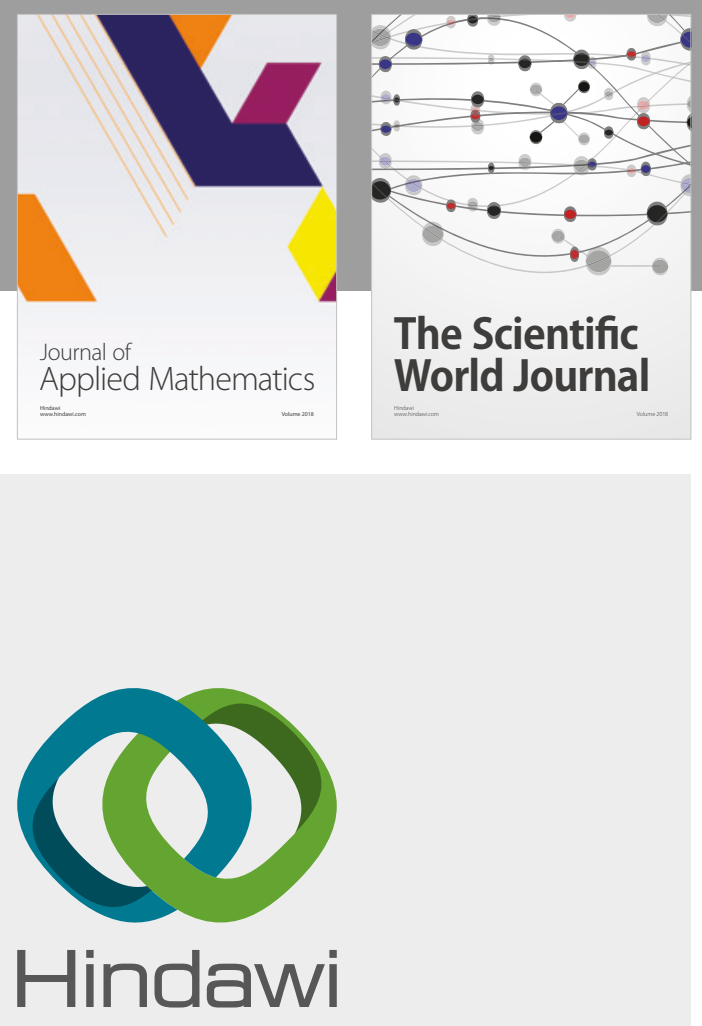

Submit your manuscripts at

www.hindawi.com

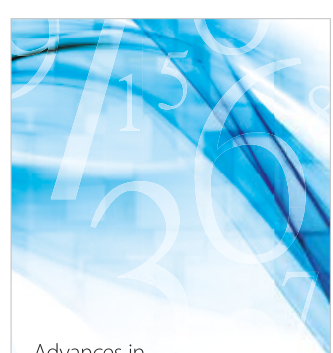

Advances in
Numerical Analysis
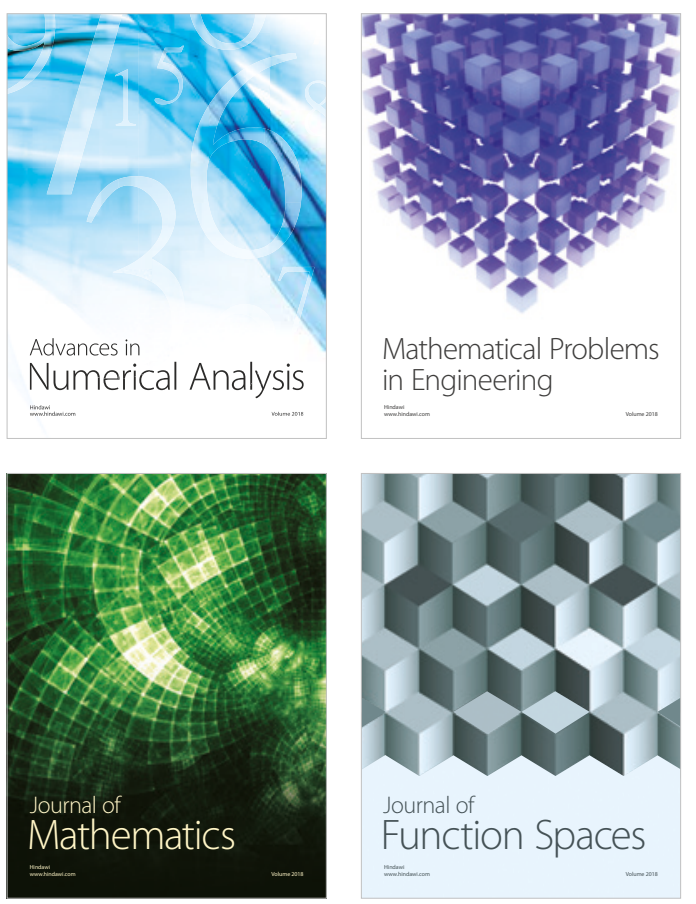

Mathematical Problems in Engineering

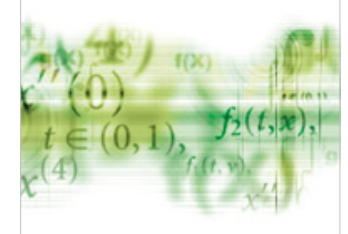

International Journal of

Differential Equations

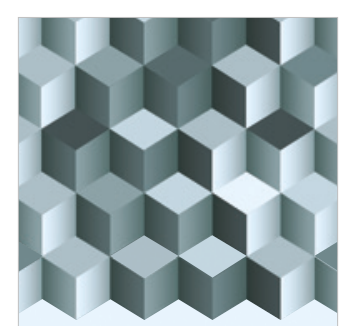

Journal of

Function Spaces
The Scientific

World Journal

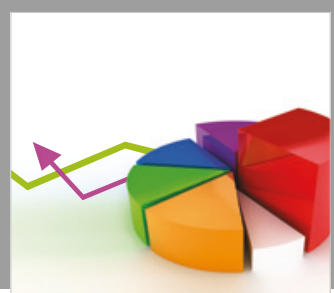

Journal of

Probability and Statistics
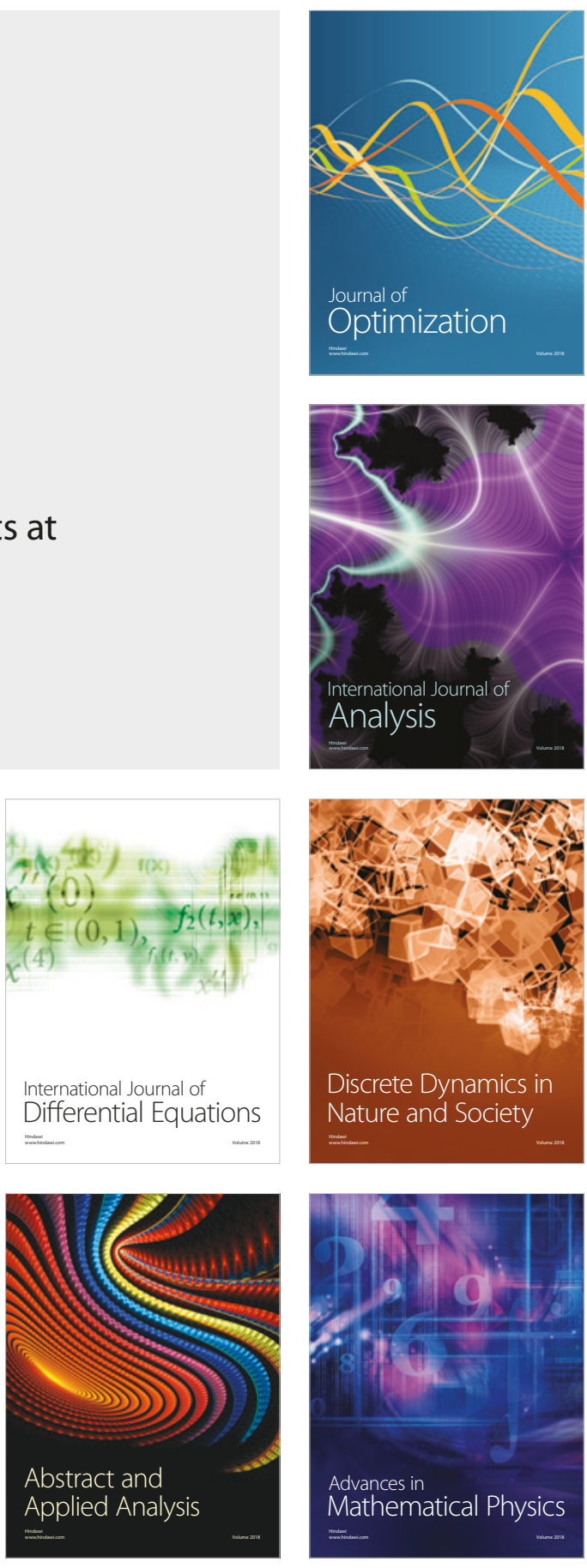